\title{
Pressure measurements on a deforming surface in response to an underwater explosion in a water-filled aluminum tube
}

\author{
G. Chambers ${ }^{\mathrm{a}}$, H. Sandusky ${ }^{\mathrm{a}}$, F. Zerilli ${ }^{\mathrm{a}}$, \\ K. Rye ${ }^{\mathrm{b}}, \mathrm{R}$. Tussing ${ }^{\mathrm{b}}$ and Jerry Forbes ${ }^{\mathrm{c}}$ \\ ${ }^{a}$ Naval Surface Warfare Center, Indian Head \\ Division, 101 Strauss Avenue, Indian Head MD \\ 20640-5035, (301) 744-2379, USA \\ ${ }^{\mathrm{b}}$ Naval Surface Warfare Center, Carderock Division, \\ Carderock MD 20817-5700, USA \\ ${ }^{\mathrm{c}}$ Lawrence Livermore National Laboratory, 7000 East \\ Avenue, Livermore, CA 94550, USA
}

Received 7 July 1998

Revised 8 February 2000

Experiments have been conducted to benchmark DYSMAS computer code calculations for the dynamic interaction of water with cylindrical structures. Small explosive charges were suspended using hypodermic needle tubing inside $\mathrm{Al}$ tubes filled with distilled water. Pressures were measured during shock loading by tourmaline crystal, carbon resistor and ytterbium foil gages bonded to the tube using a variety of adhesives. Comparable calculated and measured pressures were obtained for the explosive charges used, with some gages surviving long enough to record results after cavitation with the tube wall.

Keywords: DYSMAS, shock validation, wall deformation, underwater detonation, computer modeling, streak photography, tourmaline gage

\section{Introduction}

Computer modeling codes can provide solutions to large or complex events that are not easily tested. Since approximations to physical and chemical processes are required in the codes, they must be validated by precise experiments prior to the intended application. Of interest to this study is the complex interaction between a shock wave in water and a deformable structure, given that the properties of the explosive, water and wall are known. The problem is complex, both experimentally and numerically, because the water momentarily separates (cavitates) during the interaction. In a previous experiment (Sandusky and Chambers [3]), wall deformation and velocity were measured directly using a streak camera and a Doppler laser velocimeter. In the current study, attempts were made to measure pressure at the water/wall interface, free-field pressure, and both inner and outer-wall strain.

The Guirguis Hydro-Bulged Cylinder experiment was conceived for model validation of the DYSMAS code [1]. The radially-symmetric arrangement consists of a water-filled tube with a centrally-positioned explosive charge. The symmetrical interior loading removes the complication of bubble collapse driving a water jet towards the structure, which occurs in the usual exterior loading. Interior loading also permits easy access to the deforming structure by various optical techniques for dynamic measurements.

The experimental data have been modeled using the codes WONDY V [2], which is 1-D Lagrangian, and the code DYSMAS/C [1], which is a coupled Eulerian/Lagrangian simulation. Advantages of both Eulerian and Lagrangian scheme are incorporated into DYSMAS which is the code to be validated.

\section{Experimental arrangement}

The experimental arrangement previously used in Fig. 1 is the same as that previously (1) used, except for a shorter tube to facilitate internal gauge installation. The deformable structure was a $17.8 \mathrm{~cm}$ long by $10.2 \mathrm{~cm}$ outer diameter (O.D.) Al 5086 tube with a $0.635 \mathrm{~cm}$ wall thickness. The runout (roundness) and thickness of the tube wall varied by only $0.005 \mathrm{~cm}$. The tube was sealed at the bottom by a $0.25 \mathrm{~cm}$ thick polymethyl-methacrylate (PMMA) sheet with a scored circle corresponding to the inner diam- 


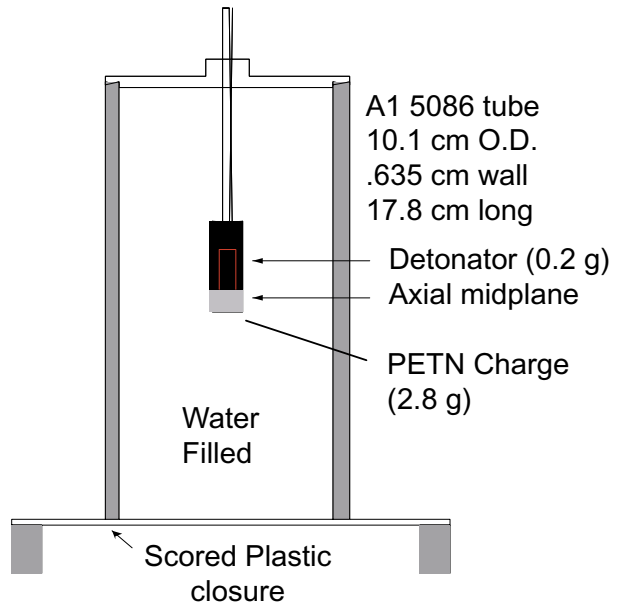

Fig. 1. Experimental arrangement.

eter of the tube. With this weakened bottom closure and the tube mostly open on top, there was little resistance to the de-oxygenated water that initially filled the tube from being ejected by the exploding charge. The charge consisted of $2.8 \mathrm{~g}$ pentaerythritol tetranitrate (PETN) pellet and an exploding bridgewire detonator with another $0.2 \mathrm{~g}$ of PETN. The detonator was encased in a PMMA sleeve of the same diameter as the explosive pellet. These components were bonded together, waterproofed, and suspended in the center of the $\mathrm{Al}$ 5086 tube with hypodermic needle tubing. The needle tubing provided a conduit for the detonator leads and was further filled with epoxy to prevent collapse during shock loading, thereby simplifying the modeling. Preliminary DYSMAS [1] calculations predicted final wall strains of $\sim 10 \%$ without rupture from detonating $3.0 \mathrm{~g}$ of PETN.

Two experiments (Shot SV-5,6) were conducted at the former White Oak Laboratory of the Naval Surface Warfare Center (NSWC), as in the previously reported work (1); and one experiment (Shot SV-7) was conducted at the Lawrence Livermore National Laboratory (LLNL). The arrangement was identical at the two laboratories, except that the explosive pellet was pure PETN in the White Oak experiments versus LX16 (96/4 PETN/inert binder) in the LLNL experiment. Since the LX-16 pellet contained the same amount of PETN, it was slightly longer than the $1.27 \mathrm{~cm}$ for the pure PETN pellets but resulted in essentially identical wall deformation, as expected. Shot SV-7 was instrumented with streak photography to obtain dynamic wall displacement and Fabry-Perot interferometry to obtain wall velocity for comparison with similar data obtained at White Oak (Sandusky and Chambers [3]). These data are not discussed so that emphasis can be given to the pressure measurements obtained at the water/wall interface in these experiments.

Various techniques were attempted to obtain reliable strain and pressure measurements inside the tube while surviving $\geqslant 100 \mu \mathrm{s}$ in a complex environment. In addition to the gauges and their mounting, consideration was given to the leads and their connection to the gauges. Protecting the leads by drilling holes in the tube wall was not attempted to avoid perturbing tube deformation; therefore, the leads passed through the water to the top of the tube and were subjected to a similar environment as the gauges.

Strain measurement with a foil gauge on the inner wall was of interest itself and for developing techniques to enhance survivability of similarly constructed pressure gauges. Previously, only the onset of deformation $(<1 \%$ strain) had been measured on the outer wall by circumferentially-oriented constantan foil and optical fiber gauges bonded with cyanoacrylate [3]. In the current experiments, some of the gauges consisted of annealed constantan foils with $20 \%$ strain capability and were bonded by an epoxy that could achieve similar strains. It was anticipated that small lead connections to the gauge tabs would avoid premature mechanical failure from the shock loading normal to the gauge and the rapid straining in the gauge plane. Either the ends of 32 AWG wires $(0.36 \mathrm{~mm}$ diameter conductor) were peened flat and soldered to the tabs or the manufacturer had electronically welded even finer magnet wire or ribbon leads to the tabs.

Pressure measurements were obtained with tourmaline, carbon resistor, carbon film, and ytterbium gauges. The tourmaline gauges were made and calibrated by personnel at the Carderock Division of NSWC experienced in the measurement of pressures from underwater explosions. These gauges are piezoelectric, consisting of a single $3.2 \mathrm{~mm}$ diameter by $\sim 1 \mathrm{~mm}$ thick crystal with conductive paint on both ends, to which leads were soldered. They are much smaller than those typically used in underwater explosion measurements, which contain multiple $6.4 \mathrm{~mm}$ diameter crystals. Small gauges were advantageous for surviving the higher shock pressures and better resolving the shorter shock durations in the current experiments. To further improve gauge resolution, the oil-filled boot typically used in underwater explosion measurements was removed, except for one gauge. Despite the small crystals, the electrical charge generation at the expected shock pressures required no amplification; gauge signals were directly connected to oscilloscope inputs with 


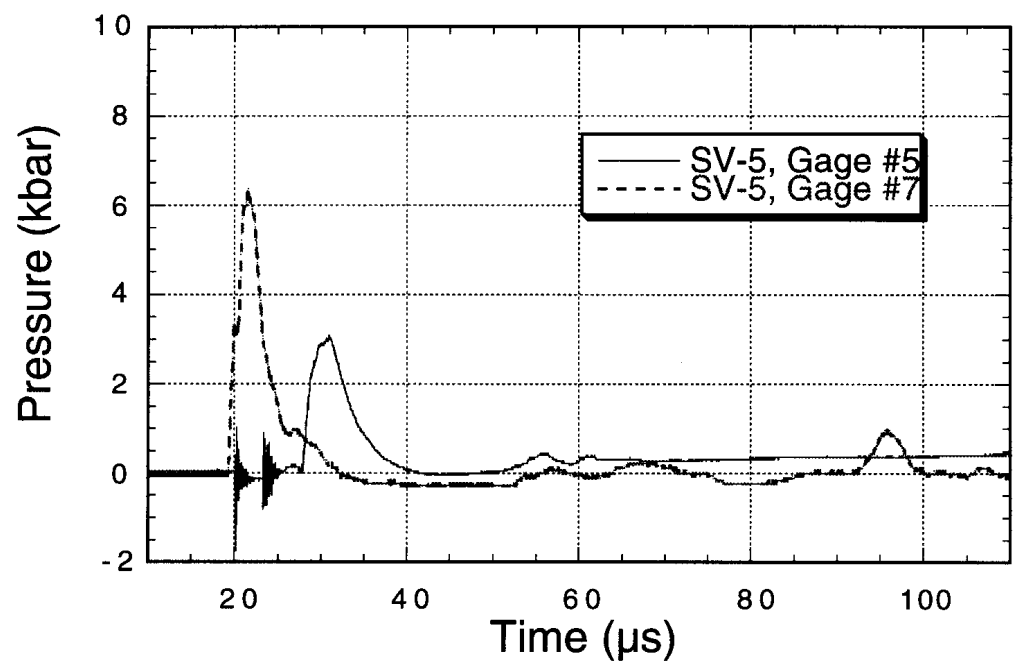

Fig. 2. Pressure from tourmaline gages at the wall/water interface in Shot SV-5.

$1 \mathrm{M} \Omega$ impedance. In Shot SV-5, seven gauges with various constructions were attempted for measurements at the axial midplane and $38 \mathrm{~mm}$ above the midplane. Four gauges simply had twin lead or 32 AWG wires soldered to the crystals. For Gauge 5, the crystal was inside a hole in a Mylar strip that had a copper ribbon on each side for leads. The crystal in Gauge 6 was connected to a RG-174 coaxial cable within an oil-filled boot. The crystal in Gauge 7 was connected to RG-174 cable and then coated with polyurethane. A gauge of similar construction was used in Shot SV-7, except that the RG-174 cable sheath was removed and then the braided shield was sealed with polyurethane. This arrangement permitted closer location of the crystal to the inner wall of the Al tube. All gauges were oriented with the crystal axis normal to the inner wall to reduce shock transit time and to enhance survivability by avoiding edge loading. Completed gauges were bonded to the inner wall within a bead of epoxy, which supported the crystal in a further attempt to improve survivability.

Carbon and ytterbium gauges are piezoresistive. The carbon gauges were either standard resistors, which are relatively rugged in a shock environment, or thin films with better resolution that is compromised some by the encapsulation required for gauge survival. Shot SV-6 was instrumented with two $470 \Omega, 1 / 8 \mathrm{~W}$ carbon resistors from the same batch as used by Wilson [4]. One gauge was located on the inner wall at the axial midplane and another was located in the water on-axis at $7.0 \mathrm{~cm}$ below the midplane. In Shot SV-6, a Dynasen FC 300-50-EKRTE carbon film gauge was also located on the inner wall at the axial midplane. This gauge had $25 \mu \mathrm{m}$ thick Kapton encapsulation, including the ribbon leads that extended beyond the top of the water, but was otherwise unarmored. A similarly located Dynasen carbon film gauge in Shot SV-7 had a $50 \Omega$ constantan strain gauge incorporated within it to correct the carbon gauge output for strain effects. This gauge was armored by further encapsulation in $25 \mu \mathrm{m}$ thickness of Teflon and then electrically shielded by wrapping it in $\mathrm{Al}$ foil. A Dynasen Yb-8-50-EK ytterbium gauge was also positioned on the inner wall at the midplane in Shot SV-7. This gauge had thicker $127 \mu \mathrm{m}$ Kapton encapsulation, which was selected to improved survivability; however, the encapsulated gauge was so stiff that it did not conform to the inner wall, except for the area of the gauge element which was clamped during bonding. All of the gauges on the inner wall were bonded to it with epoxy, except that the Al foil packet shielding the carbon gauge in Shot SV-7 was fastened with double-backed tape.

\section{Results}

All of the strain gauges in Shots SV-5,6 whether located on the inner or outer tube wall, and the carbon film gage in Shot SV-6 failed during the shock loading. These thin-film gauges were too fragile to survive at both the water/tube and tube/air interfaces. Most strain gauges were recovered with failures at lead connections.

Of the tourmaline gauges in Shot SV-5, those with wire leads did not provide reasonable records, although the crystals were recovered undamaged. Pressure-time 


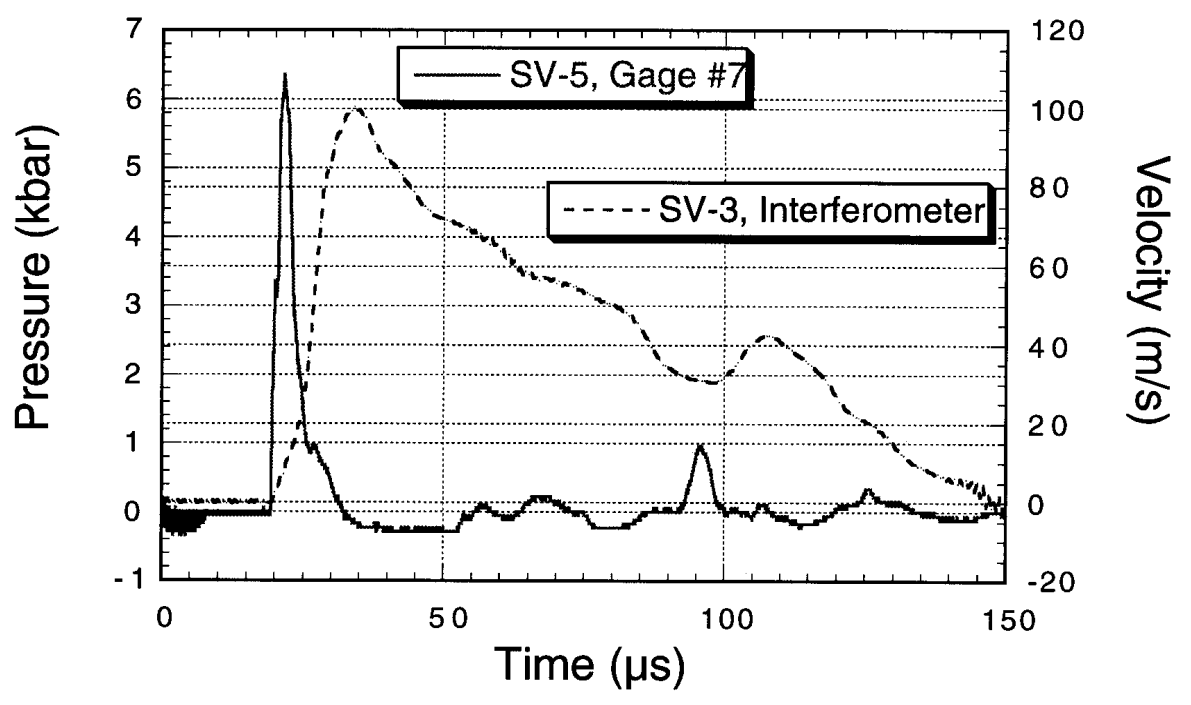

Fig. 3. Midplane comparison of pressure (Shot SV-5) and wall velocity (Shot SV-3).

histories, relative to the energizing of the detonator, from two other gauges are shown in Fig. 2. Gauge 7, mounted at the midplane on the cylinder wall, recorded an incident shock of $0.33 \mathrm{GPa}$ with a $0.8 \mu$ s risetime, a $0.5 \mu$ s plateau, and a reflected shock with a $0.8 \mu$ s risetime to a peak pressure $0.63 \mathrm{GPa}$. Separated incident and reflected shocks are observed because the crystal was mounted at least $1 \mathrm{~mm}$ from the wall. Gage 5, mounted $38 \mathrm{~mm}$ above the midplane, recorded a more diffuse wave beginning at $\sim 30 \mu \mathrm{s}$, with a peak pressure of $0.31 \mathrm{GPa}$.

Figure 3 shows a comparison at the same location of the pressure-time history from Shot SV-5, with the velocity-time profile from Shot SV-3 [1] taken from a Doppler velocimeter. Shot SV-3 was identical to Shot $\mathrm{SV}-5$ in all respects except that the Al tube was $22.9 \mathrm{~cm}$ as opposed to $17.8 \mathrm{~cm}$ long. However, differences in tube length should not affect the comparison, since rarefactions from the end of the tube have insufficient time to interact with the gages during the time frame of interest. As can be seen from Fig. 3, the initial pressure spike occurs at about $20 \mu \mathrm{s}$, causing the wall to start moving and later separate from the water. The wall velocity increases to a maximum at $35 \mu \mathrm{s}$, at which time cavitation has ended the initial shock pulse. The wall then slows down as internal forces in the Al arrest its motion. By $95 \mu \mathrm{s}$, the wall has slowed enough for the water to catch up and re-interact with it, as evidenced by the second pressure peak, followed by an immediate increase in wall velocity.

Figure 4 shows a comparison between the tourmaline pressure gage (Shot SV-5) with the carbon resis- tor gage (SV-6) and the carbon film gage (SV-7). In Shot SV-6, the carbon resistor gauge under the charge had a noisy signal, perhaps because of the unshielded loop of resistor leads that passed through the bottom closure. All gages show similar times for the incident pulse. However, only the tourmaline gage exhibits the pronounced plateau at $20 \mu \mathrm{sec}$ followed by the peak pulse $2 \mu \mathrm{sec}$ later. This is due to the positioning of this gage $\sim 1 \mathrm{~mm}$ from the wall. By contrast, the carbon gages, which were mounted directly on the wall exhibit a smooth rise, reaching their peak pressure $2 \mu$ sec after the first arrival of the shock pulse. Note that the peak width and pressure decay profiles for all three gages are similar.

Figure 5 shows a comparison of the pressure profiles of the tourmaline gage and Ytterbium gage used in Shot SV-7 with the tourmaline gage results of SV-5. The tourmaline gage in SV-7 is excessively noisy, perhaps due to capacitive pick-up on the coaxial cable lead or the lack of sheathing on the gage which may have caused the crystal to slap repeatedly against the $\mathrm{Al}$ wall. However, both tourmaline gages from the two distinct experiments reach their peak pressure at the same time, at about $3 \mu \mathrm{sec}$ after arrival of the initial shock wave. Both tourmaline gages also exhibit an initial pressure spike, followed by the second reflected wave from the wall. By contrast, the $\mathrm{Yb}$ gage peaks very quickly, within the first $\mu$ sec of the shock wave arrival. Again, the peak widths of the two tourmaline gages are virtually identical and the decay times of the pressure profiles are similar in all three gages. 


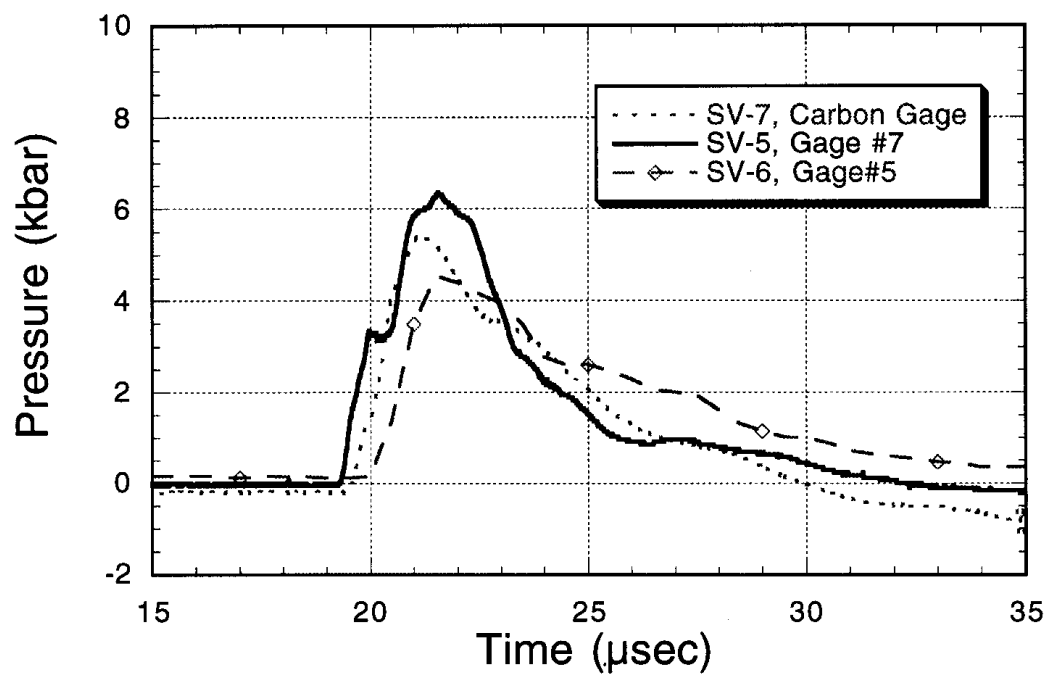

Fig. 4. Comparison of midplane from tourmaline (Shot SV-5), carbon resistor (Shot SV-6) and carbon film (Shot SV-7) gauges.

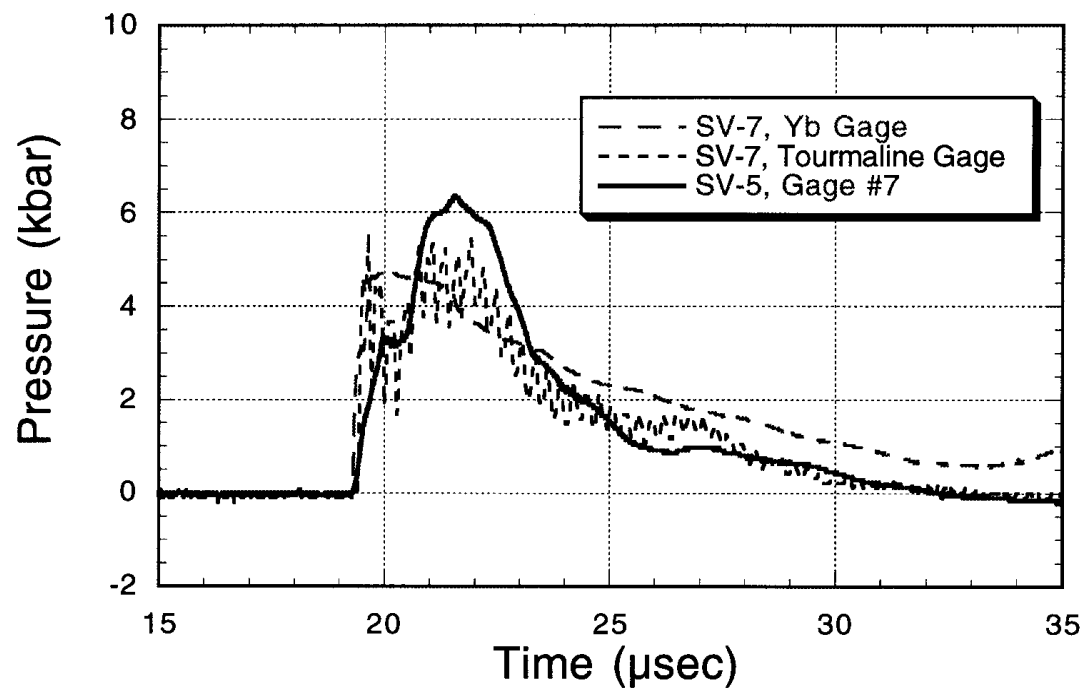

Fig. 5. Comparison of tourmaline gages from shots SV-5 and SV-7 and the Ytterbium gage from Shot SV-7.

\section{Computer modeling}

For the 1-D WONDY [2] calculations, a spherical geometry was assumed with zones of 5 and $2.8 \mu \mathrm{m}$. This is a good approximation until the shock wave reaches the cylinder wall and is sufficient to calculate the peak pressure achieved near the wall. The 2-D DYSMAS [1] calculations had uniform zones of 1 and $1.5 \mathrm{~mm}$, with a Eulerian treatment of the detonation products in water coupled to a Lagrangian treatment of the tube. Table 1 shows the standard JWL parameters for PETN used in the calculations.
Table 1

JWL parameters for PETN

\begin{tabular}{lccc}
\hline$r\left(\mathrm{~kg} / \mathrm{m}^{3}\right)$ & 1765 & $R_{1}$ & 4.4 \\
$D(\mathrm{~m} / \mathrm{s})$ & 8300 & $R_{2}$ & 1.2 \\
$A(\mathrm{GPa})$ & 617 & $w$ & 0.25 \\
$B(\mathrm{GPa})$ & 16.926 & $E_{o}\left(\mathrm{GJ} / \mathrm{m}^{3}\right)$ & 10.1 \\
\hline
\end{tabular}

The displacement of the midplane, outer diameter point calculated by DYSMAS is compared to the measured SV3 displacement in Fig. 6. The zone size had little effect on the displacement calculation. The numerical simulations underpredict the deformation compared to the deformation observed experimentally. The 


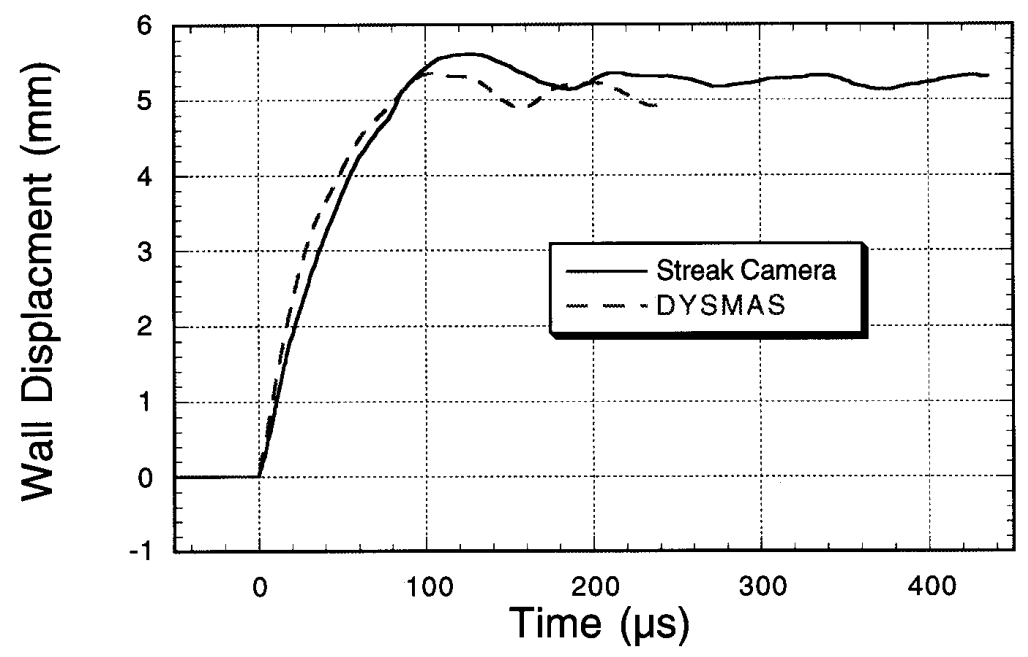

Fig. 6. Comparison of wall displacement for shot SV-3 with DYSMAS calculations.

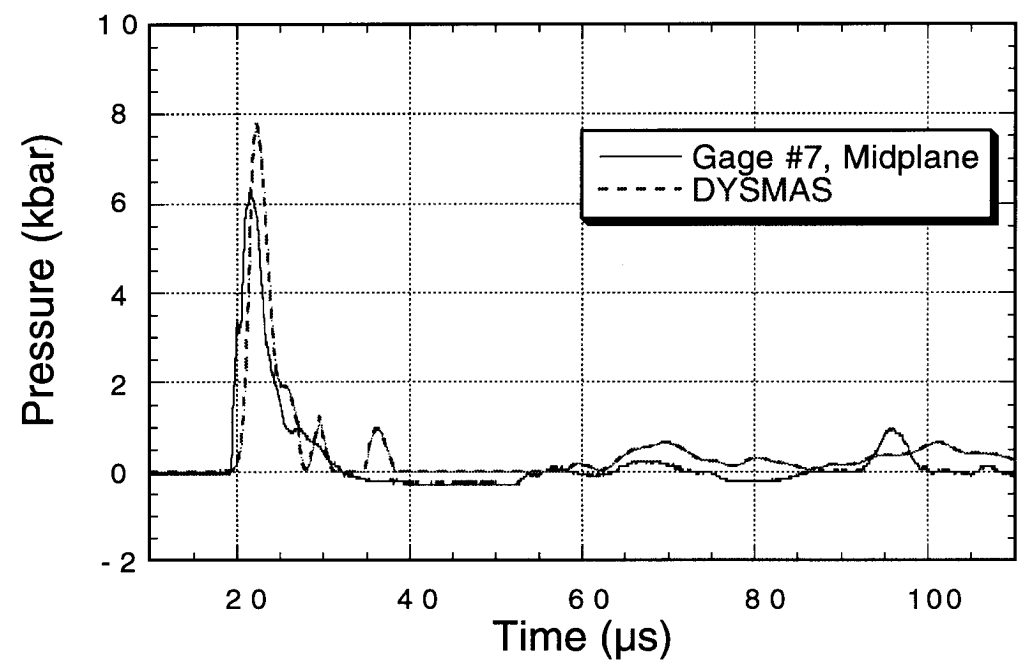

Fig. 7. Comparison of measured and calculated midplane pressures.

metal (5086 aluminum) used in the test has been well characterized. The cause for the underprediction may lie either in anisotropy in the yield strength of the 5086 due to differences in work hardening in the extrusion direction (cylinder axis) as opposed to the transverse direction or in the JWL equation of state for the explosive, PETN. It is possible that the published JWL parameters for PETN, while giving a good result for the LLNL cylinder test, may not accurately reproduce the total energy release by PETN. The displacement in the Guirguis cylinder test is relatively insensitive to the total energy. Using the equation of state described by a perturbation technique based on the intermolecular interaction of the reaction products for CHNO explo- sives we calculate the energy to be $11 \mathrm{GJ} \mathrm{m}^{-3}$ and the CJ pressure to be $28.4 \mathrm{GPa}$, compared to the published values of $10.1 \mathrm{GJ} \mathrm{m}^{-3}$ and $33.5 \mathrm{GPa}$.

The WONDY calculations predicted $0.3 \mathrm{GPa}$ incident and $0.57 \mathrm{GPa}$ reflected shock pressures in the water at the wall versus tourmaline gauge measurements of 0.35 and $0.64 \mathrm{GPa}$, respectively, thereby verifying consistency of the JWL parameters with the measurements. The midplane measurement from tourmaline gauge 7 in Shot SV-5 is shown in Fig. 7, this time compared with DYSMAS calculations at the wall/water interface for the duration of the experiment.

The code calculation for the initial shock had a $3.0 \mu \mathrm{s}$ risetime, significantly more diffuse than measured, but 
the peak pressure still exceeded the measurement by $23 \%$. The overprediction is probably caused by the nonconservative method used to reduce numerical diffusion in DYSMAS as contrasted with the underprediction to be expected when artificial viscosity is used to handle shock waves. The code calculation predicted the cavitation but excessively diffused the reloading of the wall at $\sim 90 \mu$ s following collapse of the cavitation.

\section{Summary and conclusions}

Tourmaline, carbon resistor, carbon film, and Ytterbium gages were used to measure pressures at the water/wall interface of a deforming tube in response to an underwater explosion. The consistency of the pressuretime profiles of these three different types of gages in three separate experiments validates the reproducibility of this technique. Moreover, the gauge measurements of the initial shock loading, cavitation, and reloading as cavitation collapsed corresponded to the timing of previous wall velocity measurements. Fine-mesh calculations with the 1-D WONDY code verified the pres- sure measurements until just after shock reflection at the wall, when the calculations were no longer valid. The coarser mesh 2-D DYSMAS calculations reasonably predicted the initial shock loading and cavitation, but excessively diffused the subsequent loading.

\section{Acknowledgments}

Discussions with R. Guirguis and funding from the Office of Naval Research. are gratefully acknowledged.

\section{References}

[1] Dynamic Systems Mechanics Advanced Simulation Coupled Eulerian-Langrangia Hydrocode, IAEG, Ottobrunn Germany, April 1995 version.

[2] M. Kipp and R. Lawrence, Sandia National Labs Report SAND81-0930, Alburquerque, NM, June 1982.

[3] H. Sandusky, P. Chambers, F. Zerilli, L. Fabini and L. Gottwald, 67th Shock and Vibration Symposium, Monterey CA, Nov. 1996.

[4] W. Wilson, private communication. 

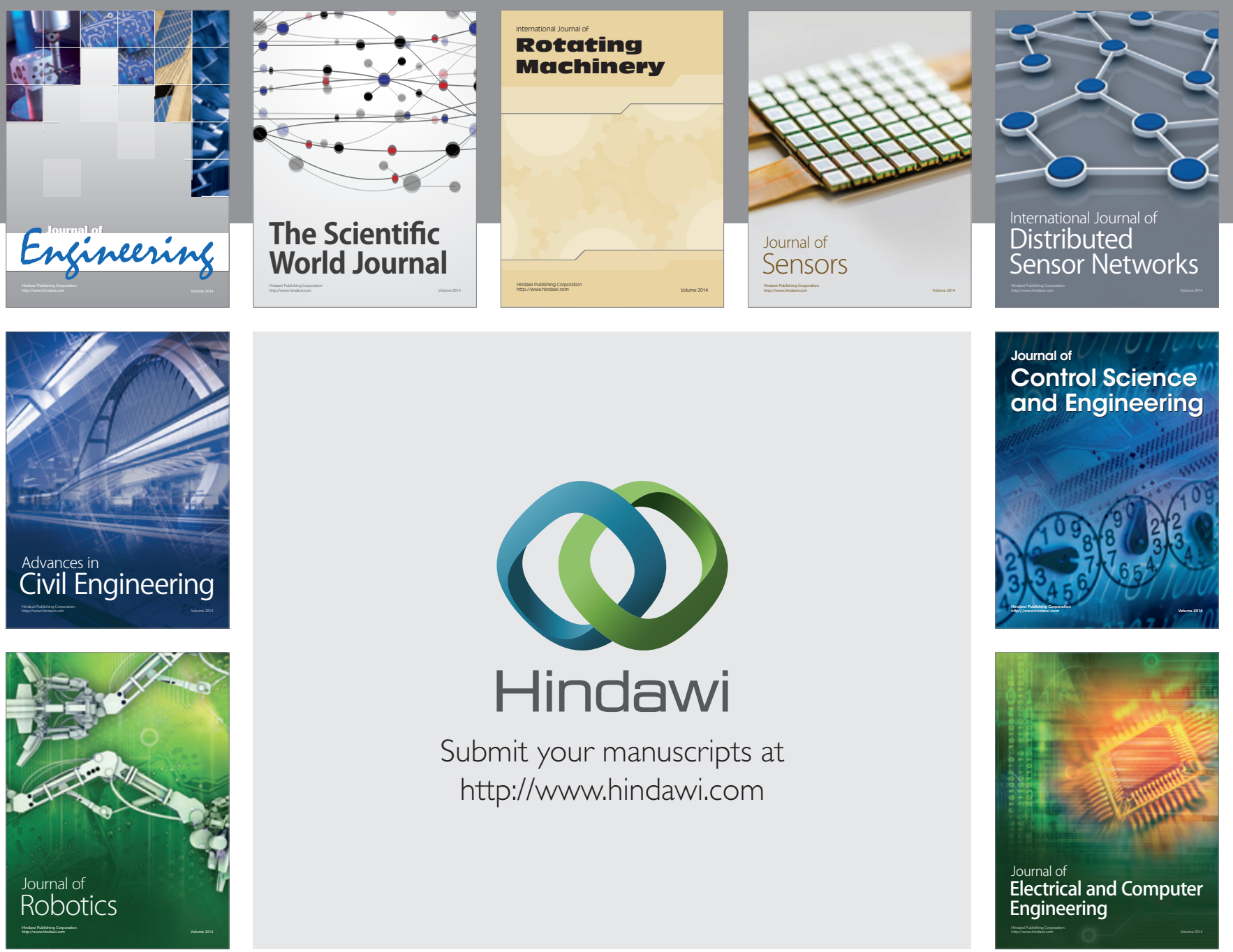

Submit your manuscripts at

http://www.hindawi.com
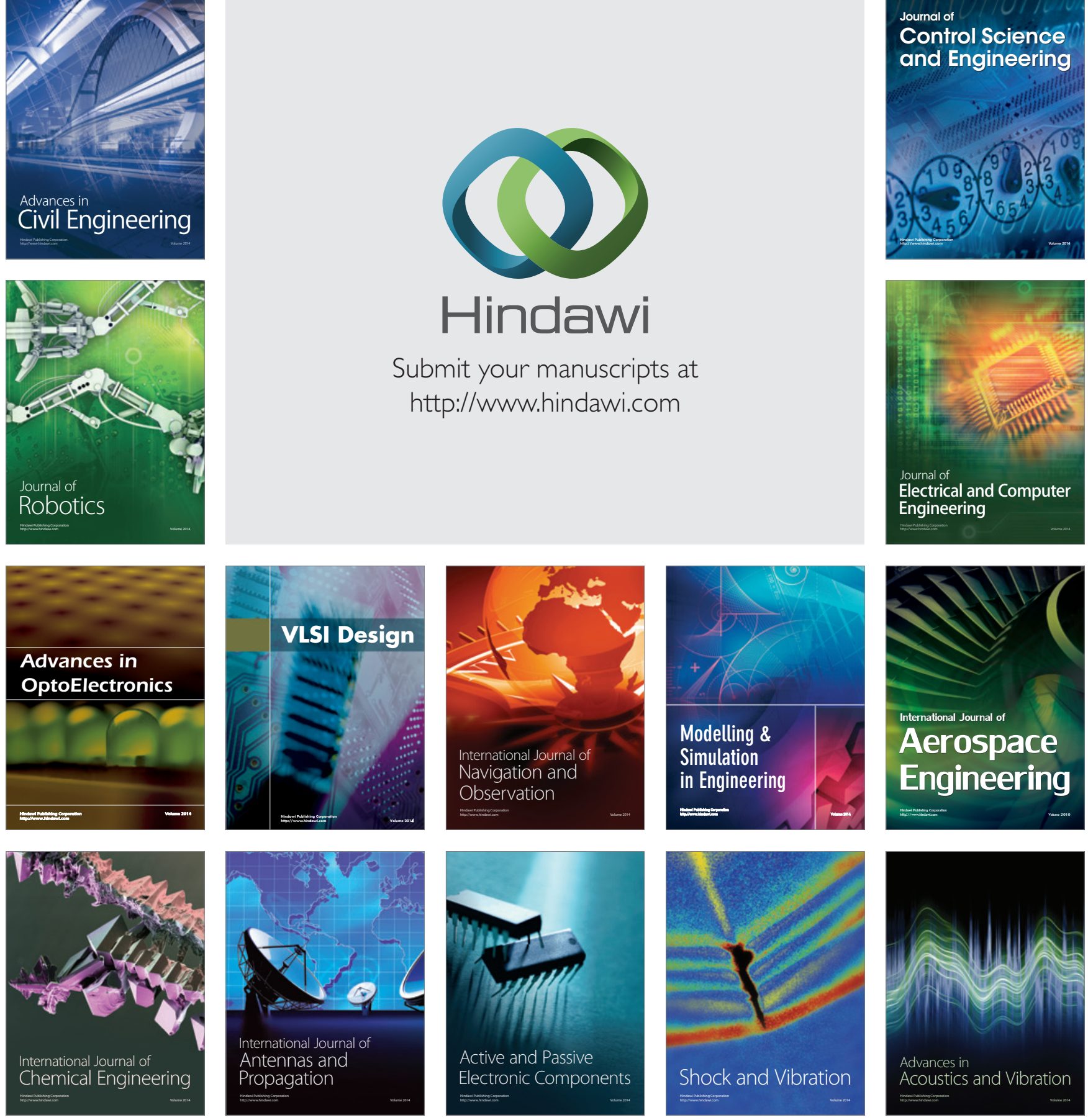